\title{
Obesidade infantil e doenças respiratórias: uma perigosa associação
}

\author{
Childhood obesity and respiratory diseases: a dangerous association
}

Obesidad infantil y enfermedades respiratorias: una asociación peligrosa

Arlete Cristina Granizo Santos ${ }^{1 *}$, Ronaldo Carvalho Santos Junior ${ }^{2}$, Isabelle Araujo de Oliveira Santana $^{1}$, Derijulie Siqueira de Sousa ${ }^{1}$, Carina Siqueira de Souza ${ }^{3}$, Carla Viviane Freitas de Jesus ${ }^{1}$, Sonia Oliveira Lima'.

\section{RESUMO}

Objetivo: Realizar uma revisão narrativa da obesidade infantil e das suas repercussões nas doenças respiratórias mais prevalentes na infância. Revisão bibliográfica: A prevalência da obesidade infantil vem aumentando progressivamente nas últimas décadas. O excesso de peso está associado a disfunções cardiometabólicas, doenças respiratórias e do sono, e muitos estudos demonstraram que é um fator evitável e modificador de alguns distúrbios, como apneia obstrutiva do sono (AOS) e asma, que acometem com muita frequência a faixa pediátrica. A relação da obesidade e AOS é bidirecional, cíclica, onde a piora de uma, reflete na outra. $O$ estado pró-inflamatório decorrente da obesidade atinge as vias aéreas, causando uma resposta inflamatória pulmonar não alérgica, assim como o efeito de acúmulo de gordura em regiões torácicas e abdominais afetam a dinâmica respiratória dificultando o controle da asma. Várias vias favorecem a liberação de citocinas devido ao excesso de ingesta de gordura, desde a microbiota intestinal, até fígado, tecido adiposo periférico e endotélio vascular. Considerações finais: Com a alta prevalência da obesidade infantil e das doenças que sofrem sua influência, adultos cada vez mais jovens estão sofrendo com distúrbios cardiovasculares, metabólicos e respiratórios.

Palavras-chave: Obesidade, Criança, Apneia obstrutiva do sono, Síndrome metabólica, Asma.

\begin{abstract}
Objective: To carry out a narrative review of childhood obesity and its repercussions on the most prevalent respiratory diseases in childhood. Bibliographic review: The prevalence of childhood obesity has been increasing progressively in recent decades. Excess weight is associated with cardiometabolic dysfunctions, respiratory diseases and sleep, and many studies have shown that it is an avoidable factor and modifier of some disorders, such as obstructive sleep apneia (OSA) and asthma, that affect the pediatric band very often. A relação da obesidade e OSA and bidirectional, cyclical, wave a piora de uma, reflect na outra. Or proinflammatory state due to obesity at the airways, causing a non-allergic pulmonary inflammation response, as well as the effect of fat accumulation in the thoracic and abdominal regions, affecting respiratory dynamics, making it difficult or controlling asthma. Several routes favor the release of cytokines due to or excess intake of fat, from intestinal microbiota, liver tissue, peripheral adipose tissue and vascular endothelium. Final considerations: With a high prevalence of childhood obesity and days that soften its influence, increasingly young adults are suffering with cardiovascular, metabolic and respiratory disorders.
\end{abstract}

Key words: Obesity, Childhood, Obstrutive sleep apneia, Metabolic syndrome, Asthma.

1 Universidade Tiradentes (Unit), Aracaju - SE. *E-mail: alertegranizo65@gmail.com

2 Universidade Federal de Sergipe (UFS), Aracaju - SE.

${ }^{3}$ Instituto Federal de Sergipe (IFS), Aracaju - SE. 


\section{RESUMEN}

Objetivo: Realizar una revisión narrativa de la obesidad infantil y sus repercusiones en las enfermedades respiratorias más prevalentes en la infancia. Revisión bibliográfica: La prevalencia de la obesidad infantil ha ido aumentando progresivamente en las últimas décadas. El exceso de peso se asocia a trastornos cardiometabólicos, respiratorios y del sueño, y muchos estudios han demostrado que es un factor evitable y modificador de algunos trastornos, como la apnea obstructiva del sueño (AOS) y el asma, que suelen afectar al grupo de edad pediátrica. La relación entre obesidad y AOS es bidireccional, cíclica, donde el empeoramiento de una se refleja en la otra. El estado proinflamatorio derivado de la obesidad llega a las vías respiratorias, provocando una respuesta inflamatoria pulmonar no alérgica, así como el efecto de la acumulación de grasa en las regiones torácica y abdominal, afectando la dinámica respiratoria, dificultando el control del asma. Varias vías favorecen la liberación de citocinas por exceso de ingesta de grasas, desde la microbiota intestinal, al hígado, tejido adiposo periférico y endotelio vascular. Consideraciones finales: Con la alta prevalencia de la obesidad infantil y las enfermedades que se ven influenciadas por ella, los adultos cada vez más jóvenes padecen trastornos cardiovasculares, metabólicos y respiratorios.

Palabras clave: Obesidad, Niño, Apnea obstructiva del sueño, Síndrome metabólico, Asma.

\section{INTRODUÇÃO}

A prevalência da obesidade no mundo vem aumentando nas últimas décadas e já é considerada uma epidemia global pela Organização Mundial de Saúde (MILLER MA, et al., 2018). A Global Nutrition Report mostrou que 40 milhões de crianças abaixo de 5 anos de idade e 330 milhões de pacientes entre 5 e 19 anos estavam com sobrepeso ou obesos em 2016 (FANZO AA, et al., 2018).

A mudança no comportamento consequente ao desenvolvimento tecnológico, fez com que as crianças permanecessem mais tempo em frente à televisão, computadores, "tablets" e celulares, especialmente as que moram em apartamentos pequenos nos grandes centros urbanos (RUNDLE AG, et al., 2020). Soma-se a isso, a influência do marketing nos hábitos alimentares das crianças, por meio de exposições de alimentos com embalagens atrativas e incentivo ao consumo de alimentos sem qualidade nutricional (LACERDA MAG, et al., 2018).

A obesidade infantil implica em diversos prejuízos, como psicológico com diminuição da autoestima, ortopédico e respiratório (SOCIEDADE BRASILEIRA DE PEDIATRIA, 2018). A persistência da obesidade da infância até a fase adulta, aumenta ainda, o risco para doenças cardiovasculares e metabólicas como diabetes mellitus tipo 2, em adultos cada vez mais jovens, o que faz dessa condição infantil um desafio para saúde pública (DI PALMO E, et al., 2021).

A privação do sono pode ocorrer por diversas causas, porém a obesidade é uma das condições atuais que mais favorecem esse distúrbio seja pela redução do tempo total de sono ou por sua fragmentação. Essas alterações também têm efeito nas funções metabólicas, endócrinas, imunológicas e da coagulação (MILLER MA, et al. 2018). A síndrome metabólica, descrita como uma constelação de riscos cardiometabólicos, é frequente nas crianças obesas, como efeito da disfunção gerada pelo acúmulo de gordura. Entretanto, se a obesidade é um fator causal importante, por outro lado, é o fator de risco mais evitável e modificador de muitas doenças respiratórias e cardiovasculares (DI PALMO E, et al., 2021).

Muitos mecanismos são sugeridos para explicar a associação entre obesidade e sintomas respiratórios, como redução da luz faríngea, maior resistência das vias aéreas, diminuição do volume pulmonar e dos fluxos aéreos, além da alteração da ventilação e trocas gasosas. A obesidade está associada com asma em 23 a $27 \%$ das crianças. A apneia obstrutiva do sono, síndrome da hipoventilação da obesidade (SHO), doença pulmonar obstrutiva crônica (DPOC), são outros exemplos de doenças respiratórias mais comuns relacionadas ao peso excessivo (LANG JE, et al., 2018).

O presente estudo objetivou realizar uma revisão narrativa das repercussões da obesidade infantil associada a doenças respiratórias na infância. 


\section{REVISÃO BIBLIOGRÁFICA}

\section{A obesidade pediátrica}

Segundo os dados da Organização Mundial da Saúde (OMS), a obesidade mundial quase triplicou nos últimos 40 anos. A prevalência do sobrepeso e obesidade entre crianças e adolescentes de 5 a 19 anos foi de 4\% em 1975 para 18\% em 2016, correspondendo a 124 milhões de crianças obesas em 2016. Em 2018, aproximadamente metade das crianças obesas menores de 5 anos, moravam na Ásia, não sendo, portanto, a obesidade infantil, um problema de saúde pública exclusivo de países desenvolvidos, ocorrendo inclusive nos centros urbanos de países subdesenvolvidos (DOOLEY AA e PILLAI DK, 2020).

Independente da classe social em que a criança está inserida, o hábito de consumo de alimentos saudáveis pode ser limitado, levando à ingestão de alimentos calóricos. Esses possuem alto teor de gorduras saturadas, carboidratos e conservantes para maior durabilidade, que favorecem o desenvolvimento da obesidade. Esses fatores associados ao estilo de vida mais sedentário contribuem para a obesidade infantil levando às repercussões danosas aos adolescentes e adultos jovens (RIBEIRO KDS, et al., 2020).

A ingestão excessiva de calorias e baixa atividade física da criança, acarreta o desenvolvimento da obesidade, que é o acúmulo de gordura corporal, principalmente na forma de ácidos graxos livres, destacando-se os órgãos sensíveis à insulina, como o fígado, tecido adiposo e músculo esquelético. A obesidade é um agravo à saúde, por induzir um processo inflamatório crônico pela liberação de radicais livres e de citocinas secretadas por adipócitos, as adipocitocinas, em especial a interleucina 6 (IL-6) e o fator de necrose tumoral alfa (TNF-alfa) (SERBIS A, et al., 2020).

Enquanto há um aumento da deposição de gordura visceral, a resistência à insulina (RI) progride, elevando os níveis séricos de ácidos graxos livres. A RI leva a um aumento da liberação desses ácidos graxos, pelo tecido adiposo e pelo fígado, com estímulo à produção de Very Low Density Lipoprotein (VLDL) e de Low Density Lipoprotein (LDL). No tecido adiposo, o excesso de gordura aumenta o fluxo de ácidos graxos livres com hipertrofia dos adipócitos, que progressivamente tornam-se resistentes à lipólise estimulada pela supressão insulínica (SERBIS A, et al., 2020).

As lipoproteínas e ácidos graxos liberados na corrente sanguínea são aterogênicos, sendo um fator de risco central nas doenças cardiovasculares. A resistência insulínica gerada pelo excesso de gordura no organismo, resulta na diminuição da captação da glicose, elevando a glicemia. O estímulo excessivo das células beta do pâncreas, produtoras de insulina, pode provocar um esgotamento da secreção dessas células, que futuramente, poderá gerar o diabetes mellitus tipo 2 (SERBIS A, et al., 2020).

A disfunção metabólica hepática, favorece o estado pró-trombótico e conjuntamente com o estado próinflamatório, a disfunção endotelial, e a aterosclerose, torna o risco cardiovascular mais iminente, se medidas não forem tomadas para quebrar esse ciclo. Esses mecanismos fisiopatológicos tornam-se cíclicos e cumulativos, e explicam como a obesidade progride para a síndrome metabólica, que é o conjunto de fatores de risco para as doenças cardiometabólicas (CRUZ JF, et al., 2019).

A leptina é produzida pelos adipócitos do tecido adiposo e agem no sistema nervoso central, ligando-se a receptores no hipotálamo, e perifericamente, em alguns órgãos, como o pâncreas. A ação da leptina é reduzir a ingestão alimentar e regular o metabolismo de glicose e gorduras. Na obesidade ocorre diminuição da sensibilidade à leptina, passando a não sinalizar à saciedade mesmo com altos níveis de gordura e carboidratos. Conforme a obesidade progride, ela vai perpetuando sua instalação, já que as adaptações do organismo, desregulam os mecanismos de controle da fome e por outro lado, desviam a homeostase para maiores níveis de gorduras plasmáticas (ROMERO CEM e ZANESCO A, 2006).

O sobrepeso e a obesidade podem ser detectados, medidos e classificados através das medidas antropométricas. A mais utilizada é o índice de massa corporal (IMC) que é o peso em kilogramas, dividido pelo quadrado da altura em metros, sendo classificada em z-escores, para faixas etárias de crianças até 5 anos de idade e para maiores de 5 anos, de acordo com a OMS (DOOLEY AA e PILLAI DK, 2020). A circunferência abdominal também tem sido utilizada como critério de obesidade central, em valores de cortes 
ou percentis. Ambas entram como um dos critérios de definição de síndrome metabólica, somando-se a marcadores plasmáticos (High Density Lipoprotein - HDL, triglicérides, glicemia e provas de resistência à insulina) e medidas de pressão arterial (SERBIS A, et al., 2020).

\section{Obesidade e Apneia Obstrutiva do Sono}

A apneia obstrutiva do sono (AOS) pertence a um espectro de distúrbios respiratórios do sono (DRS) que se caracteriza por uma obstrução recorrente parcial ou completa das vias aéreas superiores, frequentemente interrompida pelo despertar. Os DRS abrangem um grupo de transtornos que possuem padrões respiratórios anormais acompanhados ou não de ventilação insuficiente durante o sono. No caso da AOS, a interrupção da ventilação resulta em geral, em dessaturação (queda da oxigenação) e ocasionalmente em hipercapnia (DI PALMO E, et al., 2021) e é tradicionalmente associada a risco cardiovascular (BONSIGNORE MR, et al., 2019).

A obesidade é o fator causal mais significativo da AOS, sendo reportado por Verhulst SL, et al. (2008) uma prevalência de AOS em 15 a 59\% de crianças obesas, comparado à prevalência de 1 a $2 \%$ de crianças com peso dentro da normalidade. Ocorre uma deposição de gordura nas vias aéreas superiores, com estreitamento da luz, o que aumenta a área de colapsabilidade de toda a via. Soma-se a esse evento, o aumento da sobrecarga da caixa torácica, em especial na posição supina, que desequilibra o gradiente de pressão adequado para uma respiração eficiente. Além da obesidade, alterações craniofaciais e as hipertrofias adenoideanas e/ou amigdalianas, também são fatores predisponentes para AOS em crianças, e nesses casos, crianças com peso normal e baixo peso, podem ser acometidas (KOREN D, et al., 2016).

A ligação entre apneia do sono, obesidade e síndrome metabólica é muito complexa e a ocorrência simultânea entre eles, gera um ciclo vicioso de influência negativa. $O$ elo entre as três condições está nos efeitos sistêmicos desencadeados pela fragmentação do sono e pela hipóxia. A persistência do tônus simpático tanto diurno como noturno e o aumento do cortisol, induzem a elevação da pressão arterial sistêmica, arritmia e cardiopatia isquêmica, que somando-se ao efeito aterogênico da síndrome metabólica, torna-se um risco potencial cardiovascular (DI PALMO E, et al., 2021).

A hipóxia contribui para o aumento da resistência à insulina, aumento dos níveis de grelina e inflamação endotelial, exacerbando os efeitos observados também na síndrome metabólica (PILLAR G e SHEHADEH N, 2008). De acordo com Zou J, et al. (2018), a fragmentação do sono é associada a hiperinsulinemia, e a hipóxia intermitente ao Homeostasis Model Assessment - Insulin Resistance (HOMA-IR) alterado, sinais de um estado pré-diabético.

Entretanto, a principal associação entre obesidade e AOS é a leptina. Uma das ações desse peptídeo, além do controle do apetite, é estimular quimiorreceptores centrais, para desencadear a respiração. Porém, o excesso de ingesta e armazenamento de gordura leva a resistência à leptina, não permitindo que essa ação ocorra (DI PALMO E, et al., 2021). Em recentes estudos em crianças com esteatose hepática não alcoólica, observaram relação da alta ingesta de gorduras com alteração da microbiota intestinal, que desencadeia uma reação inflamatória sistêmica envolvida na obesidade e na AOS (ZHANG Y, et al., 2018).

Muitos estudos confirmam que a obesidade e AOS participam de processos semelhantes na disfunção no processo metabólico, caracterizados pelo estresse oxidativo. Assim, AOS pediátrica, como obesidade, tem sido relacionada ao aumento do risco da morbidade cardiovascular e metabólica, tanto independentemente como em sinergismo com o excesso de peso (DI PALMO E, et al., 2021).

\section{Obesidade e Asma}

A asma é uma das doenças crônicas mais frequentes entre crianças com prevalência global entre 1 e 16\%, e segundo a OMS representa $1.8 \%$ da carga global de doenças (DOOLEY AA e PILLAI DK, 2020). Embora os sintomas de asma em crianças tendem a ser mais prevalentes em países mais desenvolvidos, são mais graves em países menos influentes (LAl CKW, et al., 2009).

Normalmente a asma é vista como um processo alérgico, entretanto, já é bem estabelecida a relação da obesidade, como etiologia da asma, decorrente de um processo inflamatório não alérgico. Em crianças mais 
jovens, em torno de 3 a 4 anos de idade, a asma é um fator de risco para a obesidade (DOOLEY AA e PILLAI DK, 2020; DI PALMO E, et al., 2021), entretanto, essa relação inverte na adolescência, sendo a obesidade um fator de risco para asma (ZHANG Y, et al., 2020).

Identificou-se dois fenótipos de asma por obesidade na população pediátrica. O fenótipo de instalação tardia ocorre em pacientes com 12 anos de idade ou mais, mais frequente no sexo feminino, com quadros graves, baixo Volume expiratório forçado no primeiro segundo (FEV1) e na capacidade residual funcional (FVC), e inflamação neutrofílica/paucigranulocítica. O segundo fenótipo, ocorre em pacientes menores de 12 anos, também com quadros graves, reatividade brônquica intensa, com altos níveis de imunoglobulina $E$ (Ig E) e testes cutâneos positivos. Ambos os fenótipos não apresentam eosinofilia (HOLGUIN F, et al., 2011). Pesquisas genéticas correlacionam obesidade e asma através do polimorfismo e da pleiotropia genética, concluindo que $8 \%$ do componente genético para obesidade está presente na asma (DI PALMO E, et al., 2021).

O acúmulo de gordura na parede torácica e abdominal diminui o volume pulmonar e torácico e a excursão diafragmática, o que resulta na redução da complacência respiratória e aumento do esforço respiratório ineficiente (BONSIGNORE MR, et al., 2019; DOOLEY AA e PILLAI DK, 2020). A capacidade residual funcional e o volume de reserva expiratória são menores em pacientes obesos, como descrito por Holguin $\mathrm{F}$, et al. (2011) no fenótipo tardio, sugerindo alterações intrínsecas diferentes das que ocorrem na asma alérgica. Nos obesos há uma reatividade exacerbada das vias aéreas com aumento das taxas e do trabalho respiratórios, pouco responsivos a broncodilatadores e corticosteróides (BOULET LP, et al., 2005). Esses pacientes com excesso de peso, têm um risco aumentado de desenvolver atelectasia pulmonar periférica, aumento da pressão pulmonar, edema intersticial e hipertensão pulmonar (OOLEY AA e PILLAI DK, 2020).

Em 2005 a European Respiratory Society definiu um fenômeno que explica a redução do fluxo aéreo em obesos asmáticos, denominado disanapsis. Esse evento se caracteriza por uma desproporção entre o grau de desenvolvimento do parênquima pulmonar, com o calibre da via áerea, causando obstrução das vias aéreas com níveis normais de FEV1 (DI PALMO E, et al., 2021).

O estado pró-inflamatório da obesidade também está envolvido na diminuição da função pulmonar e nos sintomas de asma. Os marcadores inflamatórios, especialmente provenientes do tecido adiposo visceral são encontrados em níveis mais elevados em pacientes com asma. A leptina estimula a liberação de IL-6 e de TNF-alfa por macrófagos presentes no tecido adiposo, como também induz a inflamação neutrofílica brônquica. O aumento dos níveis séricos de IL-6 está associado aos sintomas mais intensos da asma (PETERS MC, et al., 2016). O quadro de células do sistema imune que compõem a asma na infância diferem, reforçando mais uma vez, a hipótese de que quando associada à obesidade difere da que ocorre em crianças não obesas. Na asma não acompanhada do excesso de peso, há uma polarização dos glóbulos brancos para células T-helper 2, enquanto em obesos para células T-helper 1, com aumento de monócitos (DI PALMO et al., 2021).

Como ocorre na AOS, a alteração do microbioma intestinal que ocorre na obesidade, aumenta a resposta inflamatória e estimula a resposta imune, com aumento da ativação de neutrófilos. Destaca-se ainda que além de serem altamente prevalentes, e semelhantes quanto à influência da obesidade, a asma e AOS estão frequentemente associadas. Os sintomas de AOS são comuns em pacientes asmáticos, que geralmente apresentam quadro de difícil controle, com redução da qualidade de vida (BONSIGNORE MR, et al., 2019).

Sugere-se ainda, em obesos, a redução de uma das 4 proteínas surfactantes, a proteína tipo A (SP-A), devido aos níveis elevados de TNF-alfa no tecido pulmonar (MASTRORILLI C, et al., 2016). A SP-A é a proteína mais abundante do surfactante correspondendo a $5 \%$ da sua massa. A alteração da composição do surfactante pode comprometer sua função, com consequente aumento na tensão alveolar, uma vez que esta substância previne o colapso alveolar durante a expiração, dificultando a expansão pulmonar. Junto a isso há um prejuízo na proteção imunológica das vias aéreas inferiores, pois ao se ligar a determinadas substâncias, especialmente carboidratos, essa proteína auxilia na eliminação de bactérias e vírus (RAMÍREZ CS e TORRETTI JT, 2004). 


\section{Intervenções para controle da obesidade infantil e doenças respiratórias}

A abordagem individual inicial e mais importante de pacientes com AOS e asma obesos, é a redução de peso. A dieta e mudanças no estilo de vida, são as mais eficientes entre os tratamentos propostos para esses pacientes com AOS (DOBROSIELSKI DA, et al., 2017).

A Sociedade Brasileira de Pediatria (2017) recomenda que as crianças mantenham os horários das refeições; que se alimentem em ambiente livre de distrações, como televisão. Deve-se evitar a oferta de guloseimas nos intervalos das refeições, recompensas ou substituição, quando houver recusa do prato servido, e incentivar a prática de atividade física sempre que possível. As atividades devem ser de preferência lúdicas com movimentos para estimular a coordenação motora, e realizadas em grupo, para consolidar as relações sociais (CARVALHO MA, et al., 2011).

É importante encorajar as crianças e os adolescentes a participarem de atividades adequadas para sua faixa etária, que sejam divertidas e deem prazer, de modo que a sua prática seja mantida por longo prazo (CARVALHO MA, et al., 2011). Para atingir essas metas, o envolvimento familiar é extremamente importante e deve ser estimulado. A alimentação saudável e atividade física em ambiente familiar, mostrou uma diminuição significativa do sobrepeso $(11,2 \%)$ e da obesidade $(7,5 \%)$, após 5 a 10 anos da intervenção (BENTO B et al., 2018).

Em vários países europeus, como Espanha, Bélgica e França, existem programas municipais de prevenção à obesidade infantil (CARVALHO MA, et al., 2011). Em estudo do programa de educação alimentar iniciado em 1992 no Norte da França, Fleurbaix-Laventie Ville Santé, observou-se que a prevalência de excesso de peso nessa região foi mais baixa, após aproximadamente dez anos do início da intervenção, concluindo que é possível reverter a prevalência elevada, através de ações comunitárias (ROMON M, et al., 2009).

Após, ou simultaneamente com a abordagem inicial do controle de peso, de acordo com a causa da AOS e da presença de comorbidades, existe opções terapêuticas para esses pacientes. Os tratamentos cirúrgicos como faringoplastias e adenoamigdalectomias são muito efetivos, porém não com $100 \%$ de eficiência, permanecendo em alguns casos, uma AOS residual (KOREN D, et al., 2016). Em casos que há contraindicações, a melhor opcão terapêutica é o uso noturno do Continuous Positive Airway Pressure (CPAP), um aparelho que gera uma pressão positiva impedindo o colabamento das vias aéreas superiores, e assim, mantendo o fluxo aéreo adequado. Entretanto a taxa de adesão a esse tratamento é insatisfatória (BONSIGNORE MR, et al., 2019).

A perda de peso em crianças asmáticas auxilia o controle da asma com medicações, com boa resposta da função pulmonar, porém não se mostrou efetiva na redução da inflamação da via aérea inferior. Observouse redução da broncoconstricção durante atividade física, em pacientes asmáticos que perderam peso. Em casos de asma neutrofílica de difícil controle, o uso de macrolídeos pode ser de grande valia. A criança sofre com as consequências da obesidade interferindo desde o aprendizado até suas relações sociais, além de favorecer processos infecciosos de vias aéreas e doenças crônicas com riscos elevados de complicações. A existência dessas afecções, independem da classe social e ocorrem especialmente nos grandes centros urbanos, com redução significativa da qualidade de vida (DI PALMO E, et al., 2021).

\section{CONSIDERAÇÕES FINAIS}

A obesidade embora seja uma doença reversível ou evitável, é uma afecção que interage de forma negativa e complexa com outras afecções, frequentemente formando um ciclo vicioso ou agindo como fator de piora. As doenças respiratórias, apneia obstrutiva do sono e asma, que atingem frequentemente as crianças, quando associadas à obesidade tornam-se de difícil controle e de maior gravidade - uma associação perigosa. A atividade física, a redução do índice de massa corpórea, melhoram a resposta medicamentosa no controle das doenças respiratórias, com redução da broncoconstricção, inclusive, em casos de doença asmática de difícil controle. A adolescência e a idade adulta serão reflexos dos hábitos do período infantil e das consequências da obesidade e de doenças respiratórias, portanto, é necessário intervir na detecção precoce dessas afecções. 


\section{REFERÊNCIAS}

1. BENTO B, et al. A higher number of school meals is associated with a less-processed diet. Jornal de pediatria, 2018; 94(4): 404-409.

2. BONSIGNORE MR, et al. Obstructive sleep apnea and comorbidities: a dangerous liaison. Multidisciplinary respiratory medicine, 2019; 14(1): 1-12.

3. BOULET LP, et al. Deep inspiration avoidance and airway response to methacholine: Influence of body mass index. Canadian respiratory journal, 2005; 12(7): 371-376.

4. CARVALHO MA, et al. Análise comparativa de métodos de abordagem da obesidade infantil. Revista Portuguesa de Saúde Pública, 2011; 29(2): 148-156.

5. CRUZ JF, et al. Sarcopenia and severity of non-alcoholic fatty liver disease. Arquivos de gastroenterologia, 2019; 56(4): 357-360.

6. DI PALMO E, et al. Childhood Obesity and Respiratory Diseases: Which Link?. Children, 2021; 8(3): 177.

7. DOBROSIELSKI DA, et al. Diet and exercise in the management of obstructive sleep apnoea and cardiovascular disease risk. European Respiratory Review, 2017; 26(144).

8. DOOLEY AA, PILLAI DK. Paediatric obesity-related asthma: Disease burden and effects on pulmonary physiology. Paediatric respiratory reviews, 2021; 37: 15-17.

9. FANZO J, et al. Global Nutrition Report: Shining a light to spur action on nutrition. 2018.

10. HOLGUIN F, et al. Obesity and asthma: an association modified by age of asthma onset. Journal of Allergy and Clinical Immunology, 2011; 127(6): 1486-1493.

11. KOREN D, et al. Impact of adenotonsillectomy on insulin resistance and lipoprotein profile in nonobese and obese children. Chest, 2016; 149(4): 999-1010.

12. LACERDA MAG, et al. A influência do marketing de alimentos nas escolhas/hábitos alimentares de crianças. International Journal of Nutrology, 2018; 11(S 01): Trab36.

13. LAI CKW, et al. Global variation in the prevalence and severity of asthma symptoms: phase three of the International Study of Asthma and Allergies in Childhood (ISAAC). Thorax, 2009; 64(6): 476-483.

14. LANG JE, et al. Being overweight or obese and the development of asthma. Pediatrics, 2018; 142(6).

15. MASTRORILLI C, et al. Asthma and allergic rhinitis in childhood: what's new. Pediatric Allergy and Immunology, 2016; 27(8): 795-803.

16. MILLER MA, et al. Sleep duration and incidence of obesity in infants, children, and adolescents: a systematic review and meta-analysis of prospective studies. Sleep, 2018; 41(4): zsy018.

17. PETERS MC, et al. Plasma interleukin-6 concentrations, metabolic dysfunction, and asthma severity: a cross-sectional analysis of two cohorts. The Lancet Respiratory Medicine, 2016; 4(7): 574-584.

18. PILLAR G, SHEHADEH N. Abdominal fat and sleep apnea: the chicken or the egg?. Diabetes care, 2008; 31(Supplement 2): S303-S309.

19. RAMÍREZ CS, TORRETTI JT. Surfactante pulmonar. Revista pediátrica electrónica. En línea(Disponible): http://www. revistapediatria. cl/vol1 num1/pdf/surfactante. pdf, 2004.

20. RIBEIRO KDS, et al. COVID-19 and Nutrition: The Need for Initiatives to Promote Healthy Eating and Prevent Obesity in Childhood. Childhood Obesity, 2020.

21. ROMERO CEM, ZANESCO A. O papel dos hormônios leptina e grelina na gênese da obesidade. Revista de Nutrição, 2006; 19(1): 85-91.

22. ROMON M, et al. Downward trends in the prevalence of childhood overweight in the setting of 12-year school-and community-based programmes. Public health nutrition, 2009; 12(10): 1735-1742.

23. RUNDLE AG, et al. COVID-19-Related School Closings and Risk of Weight Gain Among Children. Obesity, 2020.

24. SERBIS A, et al. Metabolic Syndrome in Children and Adolescents: Is There a Universally Accepted Definition? Does it Matter?. Metabolic Syndrome and Related Disorders, 2020; 18(10): 462-470.

25. SOCIEDADE BRASILEIRA DE PEDIATRIA (SBP). Sociedade Brasileira de Pediatria alerta para obesidade infantil como uma epidemia. Disponível em: https://www.sbp.com.br/imprensa/detalhe/nid/sociedade-brasileira-de-pediatriaalerta-para-obesidade-infantil-como-uma-epidemia/. Acessado em: 24 abr 2021.

26. VERHULST SL, et al. The prevalence, anatomical correlates and treatment of sleep-disordered breathing in obese children and adolescents. Sleep medicine reviews, 2008; 12(5): 339-346.

27. ZHANG Y, et al. The Dynamic Relationship Between Asthma and Obesity in Schoolchildren. American journal of epidemiology, 2020; 189(6): 583-591.

28. ZOU J, et al. Independent relationships between cardinal features of obstructive sleep apnea and glycometabolism: a cross-sectional study. Metabolism, 2018; 85: 340-347. 